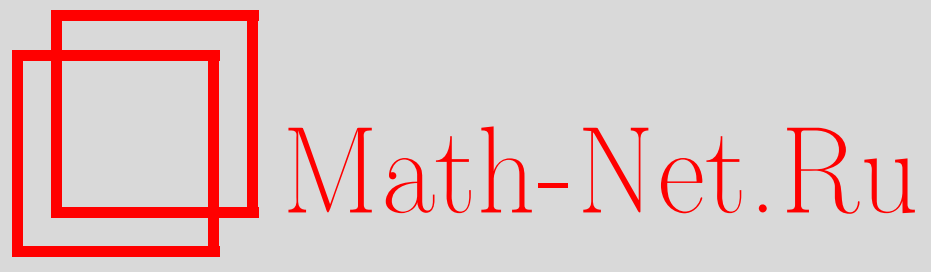

А. Е. Миронов, И. А. Тайманов, О некоторых алгебраических примерах фробениусовых многообразий, $T M \Phi$, 2007, том 151, номер 2, 195-206

DOI: https://doi.org/10.4213/tmf6039

Использование Общероссийского математического портала Math-Net.Ru подразумевает, что вы прочитали и согласны с пользовательским соглашением http: //www . mathnet.ru/rus/agreement

Параметры загрузки:

IP : 54.198 .55 .26

26 апреля 2023 г., $17: 19: 55$

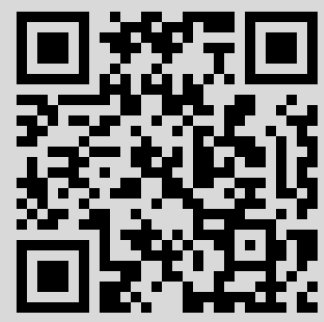




\section{О НЕКОТОРЫХ АЛГЕБРАИЧЕСКИХ ПРИМЕРАХ ФРОБЕНИУСОВЫХ МНОГООБРАЗИЙ}

\footnotetext{
С помощью аналитических методов конечнозонного интегрирования построены квазиоднородные алгебраические решения уравнений ассоциативности ВДВВ и соответствующие им неполупростые фробениусовы многообразия.
}

Ключевые слова: уравнения ВДВВ, фробениусово многообразие, метрика ДарбуЕгорова.

\section{1. ВВЕДЕНИЕ}

В этой работе мы продемонстрируем, как построить явные примеры фробениусовых многообразий с помощью аналитических методов конечнозонного интегрирования. При этом мы применим схему Кричевера для построения решений уравнений ассоциативности [1]. Хотя достаточно ясно, что решения уравнений ассоциативности, отвечающие гладким спектральным кривым, не являются квазиоднородными, мы покажем, что в сильно вырожденном случае, когда спектральная кривая состоит из рациональных неприводимых компонент, решения могут быть квазиоднородными. Расширение этих решений до фробениусовых многообразий достигается использованием технической алгебраической леммы, изложенной в разделе 5.

До недавнего времени все известные фробениусовы многообразия исчерпывались примерами Дубровина фробениусовых структур на пространствах орбит групп Кокстера (в этом случае Дубровин использовал плоскую метрику Сайто на пространстве орбит, решения уравнений Виттена-Дийкграафа-Верлинде-Верлинде (ВДВВ), отвечающие особенностям типа $A_{n}$, были найдены в [2]) и на пространствах Гурвица, квантовыми когомологиями и расширенными пространствами модулей комплексных структур на многообразиях Калаби-Яу [3]. В [4] этот список был расширен Шрамченко, “удвоившей” фробениусовы структуры Дубровина на пространствах Гурвица (многообразия Шрамченко имеют удвоенную размерность пространств Гурвица).

${ }^{*}$ Институт математики им. С. Л. Соболева СО РАН, Новосибирск, Россия. E-mail: mironov@math.nsc.ru, taimanov@math.nsc.ru 
Во всех указанных случаях многообразие с такой структурой имеет свой собственный геометрический смысл, и только квантовые когомологии могут не быть полупростыми, т.е. в касательной фробениусовой алгебре в общей точке могут содержаться нильпотентные элементы. Все наши примеры не обладают свойством полупростоты (таким образом, они не связаны напрямую с изомонодромными деформациями, см. [5]) и получаются аналитическими методами без какого-либо указания на их связи с другими геометрическими объектами. Эти примеры являются алгебраическими в том смысле, что корреляторы $c_{i j k}=\frac{\partial^{3} F}{\partial x^{i} \partial x^{j} \partial x^{k}}$ представляют собой алгебраические функции.

\section{2. НЕКОТОРЫЕ ПРЕДВАРИТЕЛЬНЫЕ ФАКТЫ О ЕГОРОВСКИХ МЕТРИКАХ И ФРОБЕНИУСОВЫХ МНОГООБРАЗИЯХ}

Для заданного симметричного тензора $\eta^{\alpha \beta}=\eta^{\beta \alpha}$ уравнения ассоциативности на функцию $F$ имеют вид

$$
\frac{\partial^{3} F(t)}{\partial t^{\alpha} \partial t^{\beta} \partial t^{\lambda}} \eta^{\lambda \mu} \frac{\partial^{3} F(t)}{\partial t^{\gamma} \partial t^{\delta} \partial t^{\mu}}=\frac{\partial^{3} F(t)}{\partial t^{\gamma} \partial t^{\beta} \partial t^{\lambda}} \eta^{\lambda \mu} \frac{\partial^{3} F(t)}{\partial t^{\alpha} \partial t^{\delta} \partial t^{\mu}},
$$

где $t=\left(t^{1}, \ldots, t^{n}\right)$, а индексы изменяются от 1 до $n$. Эти уравнения справедливы тогда и только тогда, когда конечномерная алгебра с образующими $e_{1}, \ldots, e_{n}$ и коммутативным умножением

$$
e_{\alpha} \cdot e_{\beta}=c_{\alpha \beta}^{\gamma} e_{\gamma}, \quad c_{\alpha \beta}^{\gamma}=\eta^{\gamma \delta} c_{\alpha \beta \delta}, \quad c_{\alpha \beta \gamma}=\frac{\partial^{3} F(t)}{\partial t^{\alpha} \partial t^{\beta} \partial t^{\gamma}},
$$

является ассоциативной, т.е. мы имеем

$$
\left(e_{\alpha} \cdot e_{\beta}\right) \cdot e_{\gamma}=e_{\alpha} \cdot\left(e_{\beta} \cdot e_{\gamma}\right) \quad \text { для всех } \alpha, \beta, \gamma .
$$

Уравнения (1) первоначально появились в топологической теории поля, где вместе с условиями

$$
c_{1 \alpha \beta}=\eta_{\alpha \beta}, \quad \alpha, \beta=1, \ldots, n ; \quad \eta^{\alpha \beta} \eta_{\beta \gamma}=\delta_{\gamma}^{\alpha},
$$

(метрика $\eta_{\alpha \beta}$ постоянна) и условием

$$
F\left(\lambda^{d_{1}} t^{1}, \ldots, \lambda^{d_{n}} t^{n}\right)=\lambda^{d_{F}} F\left(t^{1}, \ldots, t^{n}\right)
$$

(условием квазиоднородности) они образуют систему уравнений ВДВВ [2], [6].

Условие квазиоднородности обобщается следующим образом: предполагается, что существует векторное поле $E=\left(q_{\beta}^{\alpha} t^{\beta}+r^{\alpha}\right) \partial_{\alpha}$ такое, что $E^{\alpha} \partial_{\alpha} F=d_{F} F$ (если верно (2), мы имеем $\left.E=d_{1} t^{1} \partial_{1}+\cdots+d_{n} t^{n} \partial_{n}\right)$, и это обобщение покрывает случай квантовых когомологий.

Так как согласно [2] важно только, чтобы корреляторы $c_{i j k}$, т.е. третьи производные $F$, были квазиоднородны в смысле условия (2), существует другое обобщение квазиоднородности, которое выглядит следующим образом:

$$
E^{\alpha} \partial_{\alpha} F=d_{F} F+\text { полином второго порядка по }\left(t^{1}, \ldots, t^{n}\right) .
$$


Это обобщение важно для нас потому, что в наших примерах часть показателей $d_{i}$ равны -1 .

Геометрической формой решений уравнений ВДВВ является фробениусово многообразие, введенное Дубровиным [5], который открыл богатые дифференциальногеометрические свойства уравнений ВДВВ, что положило начало фробениусовой геометрии.

Существует важное соотношение между фробениусовыми многообразиями и егоровскими метриками, также открытое Дубровиным [7]. Метрика

$$
d s^{2}=\sum_{i=1}^{n} H_{i}^{2}(u)\left(d u^{i}\right)^{2}
$$

называется егоровской, если коэффициенты вращения $\beta_{i j}=\partial_{i} H_{j} / H_{i}, i \neq j$, являются симметричными: $\beta_{i j}=\beta_{j i}$. Рассмотрим метрики Дарбу-Егорова, т.е. плоские егоровские метрики

$$
\eta_{\alpha \beta} d x^{\alpha} d x^{\beta}=\sum_{i=1}^{n} H_{i}^{2}(u)\left(d u^{i}\right)^{2},
$$

где $x^{1}, \ldots, x^{n}$ - плоские кооординаты в некоторой области, в которой коэффициенты $\eta_{\alpha \beta}$ постоянны. Имеем

$$
\eta^{\alpha \beta}=\sum_{i} H_{i}^{-2} \frac{\partial x^{\alpha}}{\partial u^{i}} \frac{\partial x^{\beta}}{\partial u^{i}},
$$

и условие плоскости метрики вместе с симметричностью коэффициентов вращения влечет, что существует функция $F$, называемая препотенциалом, такая, что

$$
c_{\alpha \beta \gamma}=\sum_{i=1}^{n} H_{i}^{2} \frac{\partial u^{i}}{\partial x^{\alpha}} \frac{\partial u^{i}}{\partial x^{\beta}} \frac{\partial u^{i}}{\partial x^{\gamma}}=\frac{\partial^{3} F}{\partial x^{\alpha} \partial x^{\beta} \partial x^{\gamma}} ;
$$

кроме того, имеют место уравнения ассоциативности

$$
c_{\alpha \beta}^{\lambda} c_{\lambda \gamma}^{\mu}=c_{\alpha \lambda}^{\mu} c_{\beta \gamma}^{\lambda} \quad \text { для всех } \quad \alpha, \beta, \gamma=1, \ldots, n,
$$

где

$$
c_{\beta \gamma}^{\alpha}=\sum_{i} \frac{\partial x^{\alpha}}{\partial u^{i}} \frac{\partial u^{i}}{\partial x^{\beta}} \frac{\partial u^{i}}{\partial x^{\gamma}} .
$$

При условии, что ассоциативная алгебра является полупростой, обратное также верно: можно построить по решению $F(t)$ уравнений ассоциативности егоровскую метрику, удовлетворяющую (4).

\section{3. КОНЕЧНОЗОННОЕ ПОСТРОЕНИЕ ЕГОРОВСКИХ МЕТРИК И ФРОБЕНИУСОВЫХ МНОГООБРАЗИЙ}

Условие, что формула (3) определяет евклидову метрику

$$
d s^{2}=\eta_{\alpha \beta} d x^{\alpha} d x^{\beta}=\delta_{\alpha \beta} d x^{\alpha} d x^{\beta}
$$


в некоторой области (без предположения, что коэффициенты вращения симметричны) означает, что $u^{1}, \ldots, u^{n}-$ криволинейные $n$-ортогональные координаты в этой области. Это условие записывается в виде уравнений Дарбу.

Захаров первым применил методы интегрируемых систем для построения явных решений системы Дарбу [8], использовав при этом метод одевания. Впоследствии этот подход был распространен Кричевером на метод конечнозонного интегрирования.

В [9] мы применили процедуру Кричевера к сильно вырожденному случаю, когда спектральная кривая приводима и все ее неприводимые компоненты рациональны. В этом случае процедура построения решений сводится к линейным уравнениям. В этой работе мы рассмотрим спектральные кривые, удовлетворяющие таким же условиям.

Пусть Г - приводимая алгебраическая кривая такая, что все ее неприводимые компоненты $\Gamma_{1}, \ldots, \Gamma_{s}$ изоморфны $\mathbb{C} P^{1}$ и все сингулярности на $Г$ являются точками пересечений различных компонент.

Регулярный дифференциал $\Omega$ на Г задается такими мероморфными дифференциалами $\Omega_{1}, \ldots, \Omega_{s}$ на компонентах, что каждый дифференциал может иметь только простые полюсы и только в точках пересечения компонент, причем сумма вычетов в каждой точке пересечения компонент равна нулю: $\sum_{j=1}^{r} \operatorname{res}_{P} \Omega_{i_{j}}=0, P \in$ $\Gamma_{i_{1}} \cap \cdots \cap \Gamma_{i_{r}}$.

Возьмем три дивизора на Г:

$$
P=P_{1}+\cdots+P_{n}, \quad D=\gamma_{1}+\cdots+\gamma_{g_{a}+l-1}, \quad R=R_{1}+\cdots+R_{l},
$$

где $g_{a}$ - арифметический род Г. Обозначим через $k_{i}^{-1}$ некоторый локальный параметр, заданный в окрестности $P_{i}, i=1, \ldots, n$. Говорят, что $\psi\left(u^{1}, \ldots, u^{n}, z\right), z \in \Gamma$, есть функция Бейкера-Ахиезера, отвечающая данным $S=\{P, D, R\}$, если

1) $\psi e^{-u^{i} k_{i}}$ аналитична в окрестности $P_{i}, i=1, \ldots, n$;

2) $\psi$ мероморфна на $\Gamma \backslash\left\{\bigcup P_{i}\right\}$ с полюсами в $\gamma_{j}, j=1, \ldots, g_{a}+l-1$;

3) $\psi\left(u, R_{k}\right)=1, k=1, \ldots, l$.

Возьмем дополнительный дивизор $Q=Q_{1}+\cdots+Q_{n}$ на $\Gamma$ такой, что $Q_{i} \in \Gamma \backslash$ $\{P \cup D \cup R\}, i=1, \ldots, n$, и положим

$$
x^{j}\left(u^{1}, \ldots, u^{n}\right)=\psi\left(u^{1}, \ldots, u^{n}, Q_{j}\right), \quad j=1, \ldots, n .
$$

Для таких кривых с помощью схемы Кричевера получаем следующее утверждение [7].

УтВеРЖДЕНИЕ. Пусть Г допускает голоморфную инволющию $\sigma: \Gamma \rightarrow \Gamma$ такую, чmo

1) $\sigma$ имеет в точности $2 m, m \leqslant n$, неподвижных точек $P_{1}, \ldots, P_{n} \in P$ u $2 m-n$ точек из $Q$; 
2) $\sigma(Q)=Q$, т.е. точки из $Q$ либо неподвижны, либо переставляются инволюu,uей:

$$
\sigma\left(Q_{k}\right)=Q_{\sigma(k)}, \quad k=1, \ldots, n
$$

3) $\sigma\left(k_{i}^{-1}\right)=-k_{i}^{-1}$ в окрестности $P_{i}, i=1, \ldots, n$;

4) существует регулярный дифференииал $\Omega$ на Г такой, что его дивизоры нулей и полюсов имеют соответственно вид

$$
(\Omega)_{0}=D+\sigma D+P, \quad(\Omega)_{\infty}=R+\sigma R+Q
$$

Тогда $\Omega$ является поднятием некоторого мероморфного дифференциала $\Omega_{0}$ с $\Gamma_{0}=$ $\Gamma / \sigma$, ими имеем

$$
\sum_{k, l} \eta_{k l} \partial_{u^{i}} x^{k} \partial_{u^{j}} x^{l}=\varepsilon_{i}^{2} h_{i}^{2} \delta_{i j}
$$

где

$$
\begin{gathered}
h_{i}=\lim _{P \rightarrow P_{i}}\left(\psi e^{-u^{i} k_{i}}\right), \quad \eta_{k l}=\delta_{k, \sigma(l)} \operatorname{res}_{Q_{k}} \Omega_{0}, \\
\Omega_{0}=\frac{\varepsilon_{i}^{2} \lambda_{i}+O\left(\lambda_{i}\right)}{2} d \lambda_{i}, \quad \lambda_{i}=k_{i}^{-2} \quad \text { в окрестности } \quad P_{i}, \quad i=1, \ldots, n .
\end{gathered}
$$

Более того, если существует антиголоморфная инволющия $\tau: \Gamma \rightarrow \Gamma$ такая, что все неподвижные точки $\sigma$ неподвижны при $\tau, \tau^{*}(\Omega)=\bar{\Omega}$, то коэфбициенты $H_{i}(u)$ вещественны для $u^{1}, \ldots, u^{n} \in \mathbb{R}, u u^{1}, \ldots, u^{n}$ являются $n$-ортогональными координатами в плоском $n$-мерном пространстве с метрикой $\eta_{k l} d x^{k} d x^{l}$.

ДокАЗАТЕЛЬСтво этого утверждения по существу такое же, как исходное доказательство Кричевера для гладкой спектральной кривой [1]. Необходимо только рассмотреть регулярный дифференциал вместо мероморфного и вместо $g$ взять арифметический род, который отличен от геометрического в случае сингулярной кривой.

Следующая теорема выделяет специальный случай, когда эта конструкция приводит к метрикам Дарбу-Егорова и квазиоднородным решениям уравнений ассоциативности.

Теорема. 1) Пусть каждая компонента $\Gamma_{i}, i=1, \ldots, n$, содержит пару точек $P_{i}=\infty, Q_{i}=0, u k_{i}^{-1}=z_{i}$ - глобальный параметр на $\Gamma_{i}$. Предположим также, что каждая точка $a \in \Gamma_{i} \cap \Gamma_{j}$ пересечения различных компонент имеет одинаковые координаты на обеих компонентах: $z_{i}(a)=z_{j}(a)$, а инволюиия $\sigma$ на каждой компоненте имеет вид $\sigma\left(z_{i}\right)=-z_{i}$. Тогда метрика

$$
d s^{2}=\eta_{k l} d x^{k} d x^{l}=\sum_{i}\left(\varepsilon_{i}^{2} h_{i}^{2}\right)\left(d u^{i}\right)^{2}, \quad h_{i}=h_{i}\left(u^{1}, \ldots, u^{n}\right), \quad i=1, \ldots, n,
$$

построенная по этим спектральным данным, является метрикой Дарбу-Егорова. 
2) Предположим, что спектральная кривая является связной, и функиия Бейкера-Ахиезера нормирована в одной точке $r$ :

$$
\psi(u, r)=1, \quad R=r \in \Gamma .
$$

Тогда функиии

$$
c_{\alpha \beta \gamma}(x)=\sum_{i=1}^{n} H_{i}^{2} \frac{\partial u^{i}}{\partial x^{\alpha}} \frac{\partial u^{i}}{\partial x^{\beta}} \frac{\partial u^{i}}{\partial x^{\gamma}}, \quad H_{i}=\varepsilon_{i} h_{i},
$$

являются однородными:

$$
c_{\alpha \beta \gamma}\left(\lambda x^{1}, \ldots, \lambda x^{n}\right)=\frac{1}{\lambda} c_{\alpha \beta \gamma}\left(x^{1}, \ldots, x^{n}\right) .
$$

ДокАЗАтЕЛЬСтво первого утверждения теоремы следует схеме Кричевера [1]. Возьмем мероморфную функцию $f: \Gamma \rightarrow \mathbb{C}$, определенную на компонентах параметрами $z_{i}, i=1, \ldots, n$ :

$$
f(w)=z_{i}(w), \quad w \in \Gamma_{i}
$$

Тогда дифференциал

$$
\omega=f(z) \frac{\partial_{i} \psi(u, z)}{h_{i}(u)} \frac{\partial_{j} \psi(u, \sigma(z))}{h_{j}(u)}
$$

имеет полюсы только в $P_{i}$ и $P_{j}$ с вычетами $\beta_{i j}$ и $-\beta_{j i}$, соответственно, и это влечет

$$
\sum \operatorname{res} \omega=\beta_{i j}-\beta_{j i}=0
$$

Доказательство второго утверждения немедленно вытекает из следующих лемм.

Лемма 1. В условиях теоремы мы имеем равенство

$$
x^{j}\left(u^{1}+\mu, \ldots, u^{n}+\mu\right)=e^{-r \mu} x^{j}\left(u^{1}, \ldots, u^{n}\right) .
$$

ДоказАтельство. На компонентах $\Gamma_{j}$ функция $\psi$ равна

$$
\psi_{j}\left(z_{j}\right)=e^{u^{j} z_{j}}\left(f_{j 0}(u)+\frac{f_{j 1}(u)}{z_{j}-\gamma_{1}^{j}}+\cdots+\frac{f_{j k_{j}}(u)}{z_{j}-\gamma_{k_{j}}^{j}}\right) .
$$

Пусть $r \in \Gamma_{p}$. Тогда условие $\psi(r)=1$ записывается как

$$
f_{p 0}(u)+\frac{f_{p 1}(u)}{r-\gamma_{1}^{p}}+\cdots+\frac{f_{p k_{p}}(u)}{r-\gamma_{k_{p}}^{p}}=e^{-r u^{p}} .
$$

Если компоненты $\Gamma_{i}$ и $\Gamma_{j}$ пересекаются в некоторой точке $a$, то эта точка имеет одинаковые координаты на обеих компонентах, и условие $\psi_{j}(a)=\psi_{i}(a)$ принимает вид

$$
e^{a\left(u^{j}-u^{i}\right)}\left(f_{j 0}(u)+\frac{f_{j 1}(u)}{a-\gamma_{1}^{j}}+\cdots+\frac{f_{j k_{j}}(u)}{a-\gamma_{k_{j}}^{j}}\right)=\left(f_{i 0}(u)+\frac{f_{i 1}(u)}{a-\alpha_{1}^{i}}+\cdots+\frac{f_{i k_{i}}(u)}{a-\alpha_{k_{i}}^{i}}\right)
$$

Согласно (5) и (6) сдвиг $u^{j} \rightarrow u^{j}+\mu$ приводит к пропорциональному изменению коэффициентов $f_{s k}: f_{s k} \rightarrow f_{s k} e^{-r \mu}$ для всех $s, k$. Так как $x^{j}(u)=\psi_{j}(u, 0)$, это доказывает лемму. 


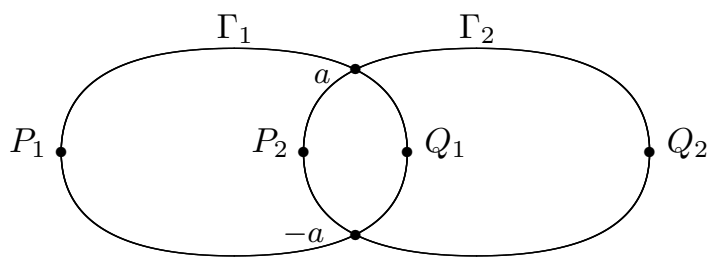

Рис. 1

Лемма 2. Имеют место равенства

$$
\frac{\partial x^{j}}{\partial u^{\alpha}}(u(\lambda x))=\lambda \frac{\partial x^{j}}{\partial u^{\alpha}}(u(x)), \quad \frac{\partial u^{\alpha}}{\partial x^{j}}(\lambda x)=\frac{1}{\lambda} \frac{\partial u^{\alpha}}{\partial x^{j}}(x) .
$$

ДокАзАтЕЛьСтво. Из леммы 1 следует, что

$$
\frac{\frac{\partial x^{j}}{\partial u^{\alpha}}\left(u^{1}+\mu, \ldots, u^{n}+\mu\right)}{x^{j}\left(u^{1}+\mu, \ldots, u^{n}+\mu\right)}=\frac{\frac{\partial x^{j}}{\partial u^{\alpha}}\left(u^{1}, \ldots, u^{n}\right)}{x^{j}\left(u^{1}, \ldots, u^{n}\right)} .
$$

Поэтому мы имеем

$$
\begin{aligned}
\frac{\partial x^{j}}{\partial u^{\alpha}}(u(\lambda x)) & =\frac{\partial x^{j}}{\partial u^{\alpha}}\left(u^{1}(x)+\mu, \ldots, u^{n}(x)+\mu\right)= \\
& =\frac{\partial x^{j}}{\partial u^{\alpha}}(u(x)) \frac{\lambda x^{j}(u(x))}{x^{j}(u(x))}=\lambda \frac{\partial x^{j}}{\partial u^{\alpha}}(u(x)), \quad \lambda=e^{-r \mu},
\end{aligned}
$$

что доказывает первое равенство. Так как $\frac{\partial u^{\alpha}}{\partial x^{j}} \frac{\partial x^{j}}{\partial u^{\beta}}=\delta_{\beta}^{\alpha}$, второе равенство вытекает из первого. Это доказывает лемму 2 и завершает доказательство теоремы.

Заданное квазиоднородное решение уравнений ассоциативности (1) с постоянной обратимой матрицей $\left(\eta^{\alpha \beta}\right)$ можно расширить до не полупростого фробениусова многообразия посредством процедуры, предложенной в разделе 5.

\section{4. ПРИМЕРЫ}

Мы приведем два примера. Первый из них является простейшим решением, входящим в бесконечное семейство, полученное в теореме. Второй пример показывает, что существуют другие решения с такими же спектральными кривыми, которые не покрываются теоремой.

ПримеР 1. Пусть Г образована двумя сферами $\Gamma_{1}$ и $\Gamma_{2}$, которые пересекаются по паре точек (см. рис. 1): $\left\{a,-a \in \Gamma_{1}\right\} \sim\left\{a,-a \in \Gamma_{2}\right\}$. Арифметический род $Г$ равен 1: $g_{a}(\Gamma)=1$.

Мы рассмотрим случай когда $n=2$ и $l=1$, т.е. функция Бейкера-Ахиезера нормирована в одной точке $r$. Положим $r \in \Gamma_{2}$ и $\psi_{2}(r)=1$. 
Функция $\psi$ принимает на компонентах $\Gamma_{1}, \Gamma_{2}$ вид

$$
\psi_{1}=e^{u^{1} z_{1}} f_{0}\left(u^{1}, u^{2}\right), \quad \psi_{2}=e^{u^{2} z_{2}}\left(g_{0}\left(u^{1}, u^{2}\right)+\frac{g_{1}\left(u^{1}, u^{2}\right)}{z_{2}-c}\right),
$$

соответственно, и условие совместности выглядит следующим образом: $\psi_{1}(a)=$ $\psi_{2}(a), \psi_{1}(-a)=\psi_{2}(-a)$. Это влечет

$$
\begin{aligned}
& \psi_{1}= e^{u^{1} z_{1}}\left(\frac{2 a(c-r) e^{a u^{1}}+(a-r) u^{2}}{(a+c)(a-r) e^{2 a u^{2}}-(a+r)(a-c) e^{2 a u^{1}}}\right), \\
& \psi_{2}=e^{u^{2} z_{2}}\left(\frac{e^{-r u^{2}}\left((a-c) e^{2 a u^{1}}+(a+c) e^{2 a u^{2}}\right)(c-r)}{(a+c)(a-r) e^{2 a u^{2}}-(a-c)(a+r) e^{2 a u^{1}}}+\right. \\
&\left.\quad+\frac{1}{z_{2}-c} \frac{\left(a^{2}-c^{2}\right)(r-c) e^{-r u^{2}}\left(e^{2 a u^{1}}-e^{2 a u^{2}}\right)}{(a+c)(r-a) e^{2 a u^{2}}+(a-c)(a+r) e^{2 a u^{1}}}\right) .
\end{aligned}
$$

Дифференциал $\Omega$ определяется дифференциалами

$$
\Omega_{1}=\frac{\beta}{z_{1}\left(z_{1}^{2}-a^{2}\right)} d z_{1}, \quad \Omega_{2}=\frac{\left(z_{2}^{2}-c^{2}\right)}{z_{2}\left(z_{2}^{2}-a^{2}\right)\left(z_{2}^{2}-r^{2}\right)} d z_{2} .
$$

Условие регулярности $\Omega$ имеет вид

$$
\operatorname{res}_{a} \Omega_{1}=\operatorname{res}_{-a} \Omega_{1}=\frac{\beta}{2 a^{2}}=-\operatorname{res}_{a} \Omega_{2}=-\operatorname{res}_{-a} \Omega_{2}=-\frac{\left(a^{2}-c^{2}\right)}{2 a^{2}\left(a^{2}-r^{2}\right)},
$$

что влечет

$$
\beta=\frac{c^{2}-a^{2}}{a^{2}-r^{2}}
$$

Для получения евклидовой метрики $\eta_{\alpha \beta}=\delta_{\alpha \beta}$ мы предполагаем, что $\varepsilon_{1}^{2}=\varepsilon_{2}^{2}$ или, эквивалентно,

откуда получаем

$$
\operatorname{res}_{Q_{1}} \Omega_{1}=-\frac{\beta}{a^{2}}=\operatorname{res}_{Q_{2}} \Omega_{2}=-\frac{c^{2}}{r^{2} a^{2}}
$$

$$
\beta=\frac{c^{2}}{r^{2}}, \quad r=\frac{a}{\sqrt{2-a^{2} / c^{2}}} .
$$

Пользуясь (7) и (8), мы выражаем $r$ через свободные параметры $a$ и $c$ :

$$
r=\frac{a}{\sqrt{2-a^{2} / c^{2}}} .
$$

Чтобы получить вещественнозначные функции $x^{1}, \ldots, x^{n}$, мы должны предположить, что $\tau^{*}(\Omega)=\bar{\Omega}$ для $\tau: z_{i} \rightarrow \bar{z}_{i}, i=1,2$. Это имеет место, когда $a^{2}, c^{2}, r^{2} \in \mathbb{R}$. Препотенциал принимает вид

$$
\begin{aligned}
F_{a, c}\left(x^{1}, x^{2}\right) & =\frac{1}{4 a c}\left(2 x_{2} \sqrt{\left(a^{2}-c^{2}\right) x_{1}^{2}+c^{2} x_{2}^{2}}+\right. \\
+ & 2 c x_{1}^{2} \ln \left(-\frac{c x_{2}+\sqrt{\left(a^{2}-c^{2}\right) x_{1}^{2}+c^{2} x_{2}^{2}}}{x_{1}}\right)-\sqrt{2 c^{2}-a^{2}}\left(x_{1}^{2}+x_{2}^{2}\right) \times \\
\times & \left.\ln \left(c^{2}\left(x_{1}^{2}-3 x_{2}^{2}\right)+a^{2}\left(x_{2}^{2}-x_{1}^{2}\right)-2 x_{2} \sqrt{2 c^{2}-a^{2}} \sqrt{\left(a^{2}-c^{2}\right) x_{1}^{2}+c^{2} x_{2}^{2}}\right)\right)
\end{aligned}
$$




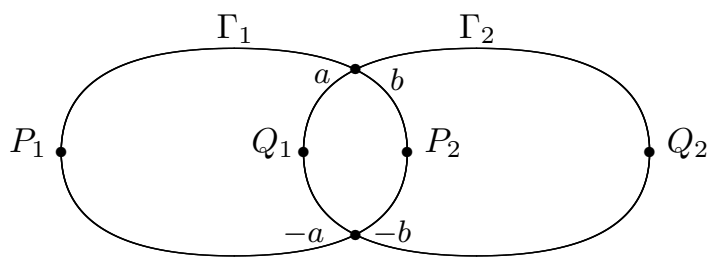

Рис. 2

и удовлетворяет уравнениям ассоциативности при $\eta_{\alpha \beta}=\delta_{\alpha \beta}$.

Когда $a=1, c=2 / \sqrt{7}$, формулы для координат и корреляторов являются достаточно простыми:

$$
\begin{aligned}
x^{1} & =\frac{4(7-\sqrt{7}) e^{u^{1}-u^{2}}}{(21-6 \sqrt{7}) e^{2 u^{1}}+(7+2 \sqrt{7}) e^{2 u^{2}}}, \\
x^{2} & =\frac{e^{-2 u^{2}\left(3(\sqrt{7}-3) e^{2 u^{1}}+(5+\sqrt{7}) e^{2 u^{2}}\right)}}{3(\sqrt{7}-2) e^{2 u^{1}}+(2+\sqrt{7}) e^{2 u^{2}}}, \\
c_{111} & =-\frac{9 x_{1}^{8}+51 x_{1}^{6} x_{2}^{2}+88 x_{1}^{4} x_{2}^{4}+\left(2 x_{1}^{2} x_{2}^{3}+4 x_{2}^{5}\right) \sqrt{\left(3 x_{1}^{2}+4 x_{2}^{2}\right)^{3}}+48 x_{1}^{2} x_{2}^{6}}{2 x_{1}\left(3 x_{1}^{4}+7 x_{1}^{2} x_{2}^{2}+4 x_{2}^{4}\right)^{2}}, \\
c_{112} & =\frac{9 x_{1}^{6} x_{2}+15 x_{1}^{4} x_{2}^{3}-8 x_{1}^{2} x_{2}^{5}+\left(2 x_{1}^{2} x_{2}^{2}+4 x_{2}^{4}\right) \sqrt{\left(3 x_{1}^{2}+4 x_{2}^{2}\right)^{3}}-16 x_{2}^{7}}{2\left(3 x_{1}^{4}+7 x_{1}^{2} x_{2}^{2}+4 x_{2}^{4}\right)^{2}}, \\
c_{122} & =-\frac{9 x_{1}^{7}+15 x_{1}^{5} x_{2}^{2}-8 x_{1}^{3} x_{2}^{4}+\left(2 x_{1}^{3} x_{2}+4 x_{1} x_{2}^{3}\right) \sqrt{\left(3 x_{1}^{2}+4 x_{2}^{2}\right)^{3}}-16 x_{1} x_{2}^{6}}{2\left(3 x_{1}^{4}+7 x_{1}^{2} x_{2}^{2}+4 x_{2}^{4}\right)^{2}}, \\
c_{222} & =\frac{-27 x_{1}^{6} x_{2}-16 x_{2}^{7}-72 x_{1}^{2} x_{2}^{5}+\left(4 x_{1}^{2} x_{2}^{2}+2 x_{1}^{4}\right) \sqrt{\left(3 x_{1}^{2}+4 x_{2}^{2}\right)^{3}}-81 x_{1}^{4} x_{2}^{3}}{2\left(3 x_{1}^{4}+7 x_{1}^{2} x_{2}^{2}+4 x_{2}^{4}\right)^{2}} .
\end{aligned}
$$

ПримеР 2. Пусть комплексная кривая Г та же, что и в примере 1 . В отличие от примера 1, мы предположим, что

$$
P_{1}=\infty \in \Gamma_{1}, \quad P_{2}=0 \in \Gamma_{1}, \quad Q_{1}=\infty \in \Gamma_{2}, \quad Q_{2}=0 \in \Gamma_{2},
$$

точка нормализации $R=r$ лежит на $\Gamma_{1}$, и дивизор полюсов $D=c$ лежит на $\Gamma_{2}$ (см. рис. 2). Вместе с тем мы не предполагаем, что точки пересечения имеют одинаковые координаты:

$$
a \sim b, \quad-a \sim-b, \quad \pm a \in \Gamma_{1}, \quad \pm b \in \Gamma_{2}, \quad a \neq b .
$$

Зададим функцию Бейкера-Ахиезера так:

$$
\psi_{1}=e^{u^{1} 2 z_{1}+\frac{u^{2}}{2 z_{1}}} f(u), \quad \psi_{2}=g_{0}(u)+\frac{g_{1}(u)}{z_{2}-c} .
$$

Дифференциал $\Omega$ определяется дифференциалами

$$
\Omega_{1}=\frac{z_{1}}{\left(z_{1}^{2}-a^{2}\right)\left(z_{1}^{2}-r^{2}\right)} d z_{1}, \quad \Omega_{2}=\frac{\left(z_{2}^{2}-c^{2}\right)}{z_{2}\left(z_{2}^{2}-b^{2}\right)} d z_{2} .
$$


Имеем условие регулярности

$$
\operatorname{res}_{a} \Omega_{1}=\operatorname{res}_{-a} \Omega_{1}=\frac{1}{2\left(a^{2}-r^{2}\right)}=-\operatorname{res}_{b} \Omega_{2}=-\operatorname{res}_{-b} \Omega_{2}=-\frac{\left(b^{2}-c^{2}\right)}{2 b^{2}}
$$

и условие евклидовости $\varepsilon_{1}^{2}=\varepsilon_{2}^{2}$, т.е.

$$
\operatorname{res}_{Q_{1}} \Omega_{2}=-1=\operatorname{res}_{Q_{2}} \Omega_{2}=\frac{c^{2}}{b^{2}} .
$$

Эти условия выполнены тогда и только тогда, когда $b= \pm i c$ и $a^{2}-r^{2}=-1 / 2$. Положим

$$
b=i, \quad c=-1, \quad a=\frac{i}{2}, \quad r=\frac{1}{2},
$$

что дает

$$
\begin{aligned}
& x^{1}=e^{-u^{1}-u^{2}}\left(\cos \left(u^{1}-u^{2}\right)+\sin \left(u^{1}-u^{2}\right)\right), \\
& x^{2}=e^{-u^{1}-u^{2}}\left(\cos \left(u^{1}-u^{2}\right)-\sin \left(u^{1}-u^{2}\right)\right) .
\end{aligned}
$$

Мы получаем метрику Дарбу-Егорова

$$
d s^{2}=\left(d x^{1}\right)^{2}+\left(d x^{2}\right)^{2}=4 e^{-2\left(u^{1}+u^{2}\right)}\left(\left(d u^{1}\right)^{2}+\left(d u^{2}\right)^{2}\right)
$$

и квазиоднородные решения уравнений ассоциативности (1) потому, что, как и в условиях теоремы, имеем

$$
x^{i}\left(u^{1}+\mu, u^{2}+\mu\right)=e^{-2 \mu} x^{i}\left(u^{1}, u^{2}\right), \quad i=1,2 .
$$

Эти решения выглядят очень просто, препотенциал $F\left(x^{1}, x^{2}\right)$ равен

$$
F\left(x^{1}, x^{2}\right)=-\frac{1}{8}\left(\left(x^{1}\right)^{2}+\left(x^{2}\right)^{2}\right) \ln \left(\left(x^{1}\right)^{2}+\left(x^{2}\right)^{2}\right) .
$$

Более того, он включен в линейное семейство квазиоднородных функций:

$$
\begin{aligned}
F_{q}\left(x^{1}, x^{2}\right)= & q\left(\left(x^{1}\right)^{2}+\left(x^{2}\right)^{2}\right) \operatorname{arctg}\left(\frac{x^{1}}{x^{2}}\right)- \\
& -\frac{1}{8}\left(\left(x^{1}\right)^{2}+\left(x^{2}\right)^{2}\right) \ln \left(\left(x^{1}\right)^{2}+\left(x^{2}\right)^{2}\right), \quad q \in \mathbb{R},
\end{aligned}
$$

которые удовлетворяют уравнениям ассоциативности при $\eta_{\alpha \beta}=\delta_{\alpha \beta}$.

Корреляторы для $F$ также выглядят очень просто:

$$
\begin{aligned}
& c_{111}=-\frac{3}{2} \frac{x^{1}}{\left(x^{1}\right)^{2}+\left(x^{2}\right)^{2}}+\frac{\left(x^{1}\right)^{3}}{\left(\left(x^{1}\right)^{2}+\left(x^{2}\right)^{2}\right)^{2}}, \\
& c_{112}=-\frac{1}{2} \frac{x^{2}}{\left(x^{1}\right)^{2}+\left(x^{2}\right)^{2}}+\frac{\left(x^{1}\right)^{2} x^{2}}{\left(\left(x^{1}\right)^{2}+\left(x^{2}\right)^{2}\right)^{2}},
\end{aligned}
$$

а формулы для $c_{122}$ и $c_{222}$ получаются из предыдущих взаимной перестановкой индексов $1 \leftrightarrow 2$. 


\section{5. АЛГЕБРАИЧЕСКАЯ ЛЕММА}

Лемма 3. Пусть $F\left(t^{1}, \ldots, t^{n}\right)$ - решение уравнений ассоииативности с постоянной метрикой $\eta_{\alpha \beta}$. Тогда функция

$$
\widetilde{F}\left(t^{0}, t^{1}, \ldots, t^{n}, t^{n+1}\right)=\frac{1}{2}\left(\eta_{\alpha \beta} t^{\alpha} t^{\beta} t^{0}+\left(t^{0}\right)^{2} t^{n+1}\right)+F\left(t^{1}, \ldots, t^{n}\right)
$$

удовлетворяет уравнениям ассочиативности (1) с метрикой

$$
\tilde{\eta}=\left(\begin{array}{lll}
0 & 0 & 1 \\
0 & \eta & 0 \\
1 & 0 & 0
\end{array}\right)
$$

ассочиативная алгебра, порожденная элементами $e_{0}, e_{1}, \ldots, e_{n}, e_{n+1}$, с законом умножения

$$
e_{i} \cdot e_{j}=c_{i j}^{k} e_{k}, \quad c_{i j}^{k}=\tilde{\eta}^{k l} \frac{\partial^{3} \widetilde{F}}{\partial t^{l} \partial t^{i} \partial t^{j}},
$$

имеет единицу е е такую, что

$$
e_{0} \cdot e_{k}=e_{k} \quad \text { для всех } \quad k=0, \ldots, n+1,
$$

и нильпотентный элемент $e_{n+1}$ такой, что

$$
e_{n+1}^{2}=0 \text {. }
$$

Более того, если $F$ квазиоднородна $u d_{\alpha}+d_{\beta}=c$ для всех $\alpha, \beta$ maких, что $\eta_{\alpha \beta} \neq 0$, то $\widetilde{F}$ также квазиоднородна $u d_{0}=d_{F}-c, d_{n+1}=2 c-d_{F}$, а значения $d_{\alpha}, \alpha=$ $1, \ldots, n$, те же, что и для $F$.

ДокАЗАТЕЛЬСТво этой леммы получается прямой проверкой.

Применяя эту процедуру к примерам из раздела 4, мы получим четырехмерное фробениусово многообразие $M$ с координатами $t^{0}, t^{2}=x^{1}, t^{2}=x^{2}, t^{3}$. Элемент $e_{0}$ является единицей, а элемент $e_{3}$ является нильпотентным в каждой касательной алгебре $T_{t} M: e_{n+1}^{2}=0$. В этих примерах имеем $d_{F}=2, d_{1}=d_{2}=1$ и, следовательно, $d_{0}=0$ и $d_{3}=2$.

Эти примеры задают двумерные деформации кольца когомологий $\mathbb{C} P^{2} \sharp \mathbb{C} P^{2}$. В самом деле, имеются стандартные образующие $e_{0}, \ldots, e_{3}$ в $H^{*}\left(\mathbb{C} P^{2} \sharp \mathbb{C} P^{2} ; \mathbb{C}\right)$ :

$$
e_{0} \in H^{0}, \quad e_{1}, e_{2} \in H^{2}, \quad e_{3} \in H^{4}, \quad e_{1}^{2}=e_{2}^{2}=e_{3}, \quad e_{1} e_{2}=0 .
$$

Справедливо тождество $d_{i}=\operatorname{deg} e_{i} / 2$. Эти деформации изменяют правила умножения для двумерных классов посредством добавления двумерных членов: $e_{i} e_{j}=$ $e_{3}+c_{i j}^{k}(t) e_{k}, i, j=1,2$.

Заметим, что в теории Зайберга-Виттена уравнения ассоциативности появились даже в более общей постановке, когда матрица $\eta$ не обязательно постоянна, а условие квазиоднородности опущено [10]. 
Благодарности. Работа поддержана РФФИ (грант № 06-01-00094а) и комплексным интеграционным проектом 2.15 СО РАН. А. Е. Миронов также поддержан грантом Президента Российской Федерации (грант МK-9651.2006.1) и Фондом содействия российской науке.

\section{Список литературы}

[1] И. М. Кричевер, Функи. анализ и его прилож., 31:1 (1997), 32-50.

[2] R. Dijkgraaf, E. Verlinde, H. Verlinde, Nucl. Phys. B, 352 (1991), 59-86.

[3] S. Barannikov, M. Kontsevich, Int. Math. Res. Notices, 1998, № 4, 201-215.

[4] V. Shramchenko, Comm. Math. Phys., 256 (2005), 635-680.

[5] B. Dubrovin, "Geometry of 2D topological field theories", Integrable Systems and Quantum Groups (Montecatini Terme, 1993), Lect. Notes in Math., 1620, Springer, Berlin, 1995, $120-348$.

[6] E. Witten, Nucl. Phys. B, 340 (1990), 281-332.

[7] B. Dubrovin, Nucl. Phys. B, 379 (1992), 627-689.

[8] V.E. Zakharov, Duke Math. J., 94 (1998), 103-139.

[9] А. Е. Миронов, И. А. Тайманов, Тр. МИАН, 255 (2006), 180-196.

[10] A. Marshakov, A. Mironov, A. Morozov, Phys. Lett. B, 389 (1996), 43-52.

Поступила в редакцию 13.10.2006 